\title{
Impaired emotional processing of chords in congenital amusia: electrophysiological and behavioral evidence
}

Article

Accepted Version

Creative Commons: Attribution-Noncommercial-No Derivative Works 4.0

Zhou, L., Liu, F., Jiang, J. and Jiang, C. (2019) Impaired emotional processing of chords in congenital amusia: electrophysiological and behavioral evidence. Brain and Cognition, 135. 103577. ISSN 0278-2626 doi: https://doi.org/10.1016/j.bandc.2019.06.001 Available at https://centaur.reading.ac.uk/84091/

It is advisable to refer to the publisher's version if you intend to cite from the work. See Guidance on citing.

To link to this article DOI: http://dx.doi.org/10.1016/j.bandc.2019.06.001

Publisher: Elsevier

All outputs in CentAUR are protected by Intellectual Property Rights law, including copyright law. Copyright and IPR is retained by the creators or other copyright holders. Terms and conditions for use of this material are defined in the End User Agreement.

www.reading.ac.uk/centaur 
Central Archive at the University of Reading

Reading's research outputs online 


\title{
Impaired emotional processing of chords in congenital amusia: Electrophysiological and behavioral evidence
}

\author{
Linshu Zhou ${ }^{\mathrm{a}}$, Fang Liu ${ }^{\mathrm{b}}$, Jun Jiang ${ }^{\mathrm{a}}$, Cunmei Jiang ${ }^{\mathrm{a}^{*}}$ \\ ${ }^{a}$ Music College, Shanghai Normal University, Shanghai, China \\ ${ }^{a}$ School of Psychology and Clinical Language Sciences, University of Reading, Reading, \\ $U K$
}

${ }^{*}$ Corresponding author at: Music College, Shanghai Normal University, 100 Guilin Road, Xuhui District, Shanghai 200234, China.

E-mail addresses: cunmeijiang@126.com (C. Jiang) 


\section{Abstract}

This study investigated whether individuals with congenital amusia, a neurogenetic disorder of musical pitch perception, were able to process musical emotions in single chords either automatically or consciously. In Experiments 1 and 2, we used a cross-modal affective priming paradigm to elicit automatic emotional processing through ERPs, in which target facial expressions were preceded by either affectively congruent or incongruent chords with a stimulus onset asynchrony (SOA) of $200 \mathrm{msec}$. Results revealed automatic emotional processing of major/minor triads (Experiment 1) and consonant/dissonant chords (Experiment 2) in controls, who showed longer reaction times and increased N400 for incongruent than congruent trials, while amusics failed to exhibit such a priming effect at both behavioral and electrophysiological levels. In Experiment 3, we further examined conscious emotional evaluation of the same chords in amusia. Results showed that amusics were unable to consciously differentiate the emotions conveyed by major and minor chords and by consonant and dissonant chords, as compared with controls. These findings suggest the impairment in automatic and conscious emotional processing of music in amusia. The implications of these findings in relation to musical emotional processing are discussed. (No more than 200 words)

Keywords: Congenital amusia; automatic processing; conscious processing; music emotion; affective priming; N400 


\section{Introduction}

Congenital amusia (hereafter amusia) refers to a neurodevelopmental disorder of pitch perception and production, which cannot be explained by hearing loss, intellectual deficiency, or lack of exposure to music in early life (Ayotte et al., 2002; Peretz et al., 2002). Individuals with amusia (hereafter amusics) exhibit deficits in processing pitch-related information, particularly in discriminating fine-grained musical pitch (Foxton et al., 2004; Hyde \& Peretz, 2004; Jiang et al., 2011) and pitch direction (Foxton et al., 2004; Jiang et al., 2010; Liu et al., 2010). While amusics show intact implicit processing of melodic pitch expectations (Omigie et al., 2012) and harmonic (Tillmann et al., 2012) and tonal structures (Tillmann et al., 2016; Zendel et al., 2015), they are unable to process these musical structures in an explicit manner (Jiang et al., 2016; Omigie et al., 2012; Tillmann et al., 2016; Zendel et al., 2015). Apart from musical deficits, amusics also exhibit impaired pitch perception in speech (Foxton et al., 2004; Jiang et al., 2012; Jiang et al., 2010; Liu et al., 2010; Loui et al., 2008; Patel et al., 2008), and reduced ability to identify emotional speech prosody (Thompson et al., 2012), especially when high frequency information in speech was removed (Lolli et al., 2015).

One aspect of music listening is to decode the emotional content of music (Juslin \& Laukka, 2004). Previous research has produced mixed results on whether musical deficits in amusia would lead to impaired emotional processing of music. In studies using questionnaires and experience sampling, some amusics reported musical engagement and appreciation at a similar level as controls (McDonald \& Stewart, 2008; Omigie, Mullensiefen, \& Stewart, 2012). This is consistent with the finding that most amusics were able to 
recognize musical emotions based on acoustic cues such as tempo (Gosselin et al., 2015). Furthermore, amusics showed sensitivity to the consonance-dissonance distinction in dyads of piano timbre, and also exhibited some sensitivity to the happiness-major and sadness-minor associations in triads of piano timbre (Marin et al., 2015).

However, other studies found that amusics, as compared with controls, failed to distinguish between consonance and dissonance in melodies (Ayotte et al., 2002) and chords (Cousineau et al., 2012), by providing similar pleasantness/preference ratings across consonant and dissonant versions. In addition, amusics also showed reduced sensitivity to musical emotions (happy versus sad) conveyed by mode (Gosselin et al., 2015), and failed to detect the difference in tension between Western and Indian melodies (Jiang et al., 2017).

Taken together, the above-mentioned studies suggest that amusics may or may not show impaired conscious processing of musical emotions across different types of stimuli. It remains to be examined whether amusia is associated with impairments in automatic processing of musical emotions. This warrants further investigation because research has shown that conscious and automatic emotional processing are two distinct mental processes, with the former representing a slow and limited-capacity attention mechanism and the latter a relatively fast, unconditional, and stimulus-driven process (Bargh et al., 1992; Bargh \& Chartrand, 1999; Neely, 1977; Posner \& Synder, 1975; Scherer, 2001). More importantly, there may be dissociation between early automatic and late conscious emotional processing (e.g., Castner et al., 2007; Kamio et al., 2006; Padovan et al., 2002; Suslow et al., 2003). Namely, impaired conscious emotional processing does not imply impaired automatic 
conscious emotional processing, and vice versa. For example, although Parkinson's patients show abnormal performance on conscious emotion evaluation, their automatic affective priming is unimpaired (Castner et al., 2007). In contrast, although individuals with high-functioning pervasive developmental disorders show impaired automatic affective priming of emotional faces, they are able to identify facial expressions at a conscious level (Kamio et al., 2006). This dissociation may result from the distinct neural mechanisms involved in conscious and automatic processing (e.g., Rossell et al., 2003; Gold et al., 2006). Therefore, although conscious processing of musical emotions may be impaired in amusia, it remains an open question whether automatic processing of musical emotions is impaired or preserved in amusia.

Unlike conscious processing, automatic processing has a rapid onset and decay (Collins \& Loftus, 1975; Posner \& Synder, 1975). Affective priming with stimulus onset asynchronies (SOAs) at or below $200 \mathrm{msec}$ thus provides an ideal paradigm for examining the early, automatic stage of emotion processing. According to spreading activation, a theoretical account of affective priming, a prime pre-activates the representations of affectively related targets by spreading the activation through the conceptual network and thus facilitating the encoding of targets that are congruent to the prime (Collins \& Loftus, 1975). Since consciousness requires at least $300 \mathrm{msec}$ to develop and to influence responses in priming tasks (Posner \& Synder, 1975), only in cases of very short SOAs can automatic processes be assessed. Indeed, previous studies have confirmed that affective priming with an SOA of 200 
msec reflects automatic, rather than conscious, emotional processing (Hermans et al., 2001; Hermans et al., 1994; Herring et al., 2013).

The present study aimed to investigate automatic and conscious emotional processing of isolated chords in amusia. First, two event-related potentials (ERP) experiments were designed to investigate the neural signature of automatic processing of musical emotions in amusia with a cross-modal affective priming paradigm, in which isolated chords served as primes and facial images as targets. We manipulated the construction of pitch intervals in chords that led to different emotional connotations. In Experiment 1, we used major and minor triads as primes, with both types of triads being consonant. From the perspective of music theory (Plomp \& Levelt, 1965), consonant intervals consist of tones with simple frequency ratios, whereas dissonant intervals are made of tones with complex frequency ratios. Thus, the difference between consonant and dissonant chords may be more salient than the difference between two consonant chords. To reduce task difficulty, in Experiment 2, we used consonant (triads) and dissonant chords (non-triads) as primes. Given that the modulation of the N400 (a negativity that peaks around $400 \mathrm{msec}$ after the target onset) has been suggested to reflect the affective priming effect (Goerlich et al., 2011; Goerlich et al., 2012; Kamiyama et al., 2013; Steinbeis \& Koelsch, 2008, 2011), we focused on the N400 effect in these two experiments. Second, given that automatic and conscious processing involve distinct neural mechanisms (e.g., Rossell et al., 2003; Gold et al., 2006), in Experiment 3, we further examined amusics' conscious emotional evaluation of the chords used in Experiments 1 and 2. It was predicted that isolated major/minor (Experiment 1) and 
consonant/dissonant chords (Experiment 2) would show a priming effect on emotional processing of faces in controls, but not in amusics. Moreover, we expected that amusics would also exhibit impairments in conscious emotional evaluation of those same chords as compared with controls in Experiment 3.

\section{Experiment 1: Automatic affective priming by major and minor}

\section{triads}

Major and minor triads are considered to be consonant and represent the prototypes or anchor points on which tonality induction is based (Brattico et al., 2009; Krumhansl, 2001). Because only major and minor triads can be used as a tonic in tonal music, the simple presence of a major or minor triad can be considered as a brief presentation of musical mode (Steinbeis \& Koelsch, 2011). Evidence has shown that the major mode is associated with the communication of happiness and the minor mode with sadness (e.g., Gagnon \& Peretz, 2003; Gosselin et al., 2015; Hevner, 1935). Even for isolated chords (a major or minor triad), the major-happiness and minor-sadness associations have also been demonstrated in both musically trained and untrained individuals (Steinbeis \& Koelsch, 2011).

Thus, the present experiment investigated whether the emotional connotations of major and minor triads would prime the processing of emotional faces in amusia. With a cross-modal affective priming paradigm, major/minor triads were used as prime stimuli and happy/sad facial expressions as target stimuli. Prime and target stimuli were either affectively congruent or incongruent to each other, with an SOA of $200 \mathrm{msec}$. We expected that the 
affective priming effect of major/minor triads on emotional recognition of facial expressions would be absent in amusics at both electrophysiological and behavioral levels as compared with controls.

\subsection{Method}

\subsubsection{Participants}

A total of 200 participants were recruited through advertisements in the bulletin board systems of universities in Shanghai. Before the ERP experiments, all participants completed the Montreal Battery of Evaluation of Amusia (MBEA; Peretz et al., 2003) as a measure of their musical abilities. Participants were classified as amusic if they scored 65 or under on the composite score of the three pitch-based subtests or below $78 \%$ correct on the MBEA global score, which corresponds to two standard deviations below the mean score of controls (Liu et al., 2010; Peretz et al., 2003). We also calculated the d' of the MBEA scores of all participants, and the d' of each amusic participant was below the cutoff of 1.23 reported by previous studies (Henry \& McAuley, 2013; Pfeifer \& Hamann, 2015), confirming the diagnosis of amusia in these participants with this measure.

Sixteen amusics and 16 matched controls took part in the ERP experiments. Table 1 shows the demographic information, the mean scores of the MBEA, and the pitch discrimination thresholds of the participants. As can be seen, the two groups differed significantly in the scores of the MBEA and in pitch thresholds, but were matched on age and years of education. All participants were native speakers of Mandarin, right-handed, with 
normal hearing, normal or corrected-to-normal vision, and no history of psychiatric or neurological diseases. None of them had received any extracurricular training in music. Hand dominance was assessed by the Edinburgh Handedness Inventory (Oldfield, 1971). Ethical approval was obtained from Shanghai Normal University, and all participants signed a written consent form before the experiments were conducted.

Insert Table 1, about here.

\subsubsection{Stimuli}

The prime stimuli consisted of 48 chords of $800 \mathrm{msec}$ long, of which 24 were major chords and 24 were minor chords. Both the major and minor triads, in root position and inverted six-four chords, were played in each of the 12 keys of the chromatic scale, resulting in 24 chords in each emotional category. Chords were created spanning an octave from G3 to F\#4. Sound files were created in Cubase (Steinberg Media Technologies GmbH, Hamburg, Germany), exported with the piano timbre and modified using Audition 6.0 (sampling rate $=$ 44.1 kHz, 16-bit resolution).

To verify whether the major and minor triads differed in psychoacoustic parameters of consonance, we computed the roughness and harmonicity characteristics of these chords. The calculation of roughness was based on the modified model of Vassilakis $(2001 ; 2005)$, which is consistent with the study by Vassilakis (2007)

(http://musicalgorithms.ewu.edu/algorithms/roughness.html). Following previous studies 
(Cousineau et al., 2012; McDermott, Lehr, \& Oxenham, 2010), harmonicity was evaluated by harmonics-to-noise ratio (HNR), which was calculated using Praat (http://www.fon.hum.uva.nl/praat/). Although the results showed that major $(M=45.13, S D=$ 7.24) and minor chords $(M=44.41, S D=6.85)$ did not differ in roughness $(z=-0.11, p$ $=.922, r=-.02)$, the HNRs of major chords $(M=11.29, S D=8.76)$ were significantly higher than those of minor chords $(M=4.97, S D=3.15, z=-3.23, p=.001, r=-.47)$. This finding indicates that the major and minor triads we used differed in harmonicity but not in roughness.

In order to assess the emotional connotations of these triads, eight non-musicians who did not participate in the subsequent ERP experiment were asked to rate each chord with regard to perceived happiness on a 7 -point scale $(7=$ very happy to $1=$ very sad $)$. They were also required to rate each chord with regard to perceived pleasantness on a 7 -point scale $(7=$ very pleasant to $1=$ very unpleasant). Paired samples $t$-tests showed that major chords $(M=$ $4.42, S D=1.53)$ received significantly higher ratings than minor chords $(M=3.86, S D=1.63)$ in perceived happiness $\left[t_{(7)}=4.96, p=.002\right]$. However, the ratings in perceived pleasantness did not differ significantly between major $(M=4.43, S D=0.64)$ and minor chords $[M=4.32$, $\left.S D=0.52 ; t_{(7)}=0.75, p=.48\right]$. These findings indicate that these major and minor triads were different in the happiness rating but not in the pleasantness rating.

Experimental target images comprised 24 images with happy expressions and 24 images with sad expressions, selected from the Chinese Facial Affective Picture System (CFAPS) (Gong et al., 2011). Each chord was played twice, once followed by a happy target image, 
and the second time by a sad target image. This resulted in four experimental conditions: congruent and incongruent conditions for happy chords as well as congruent and incongruent conditions for sad chords (see Figure 1). There were 96 trials in total, with 24 happy congruent trials (happy chords and happy faces), 24 happy incongruent trials (happy chords and sad faces), 24 sad congruent trials (sad chords and sad faces), and 24 sad incongruent trials (sad chords and happy faces).

Insert Figure 1, about here.

\subsubsection{Procedure}

The 96 trials were presented pseudorandomly in two blocks of 48 trials. The prime chords were presented through two loudspeakers positioned to the left and right of the participant. Visual targets appeared $200 \mathrm{msec}$ following the onset of the chord on the screen. Participants were instructed to decide as quickly and accurately as possible whether the facial expression was happy or sad by pressing one of the two response buttons. Once the participants made the responses, the presentation of both prime and target stimuli would be terminated, whether the chords lasted $800 \mathrm{msec}$ or not. Therefore, the duration of presentation of target faces depended on how quickly the participants made their responses. Following the responses, a row of XXXX was presented on the computer screen for $1500 \mathrm{msec}$ to give the participants a chance to blink until the next trial began with the appearance of a fixation point. 
The side (left or right hand) of the response was balanced across participants. The first block began with a practice session of two trials to familiarize the participants with the procedure.

\subsubsection{Electroencephalogram (EEG) recording and analysis}

EEG data were recorded from 64 standard scalp locations (International 10-20 system), digitized at a rate of $500 \mathrm{~Hz}$, with a $0.05 \mathrm{~Hz}$ low cutoff filter and a $100 \mathrm{~Hz}$ high cutoff filter. The data were referenced offline to the algebraical mean of left and right mastoid electrodes, filtered offline with a band-pass filter of 0.1 to $30 \mathrm{~Hz}$ (24-dB/oct slope). Trials were averaged offline with an epoch length of $1200 \mathrm{msec}$, including a baseline from $200 \mathrm{msec}$ to $0 \mathrm{msec}$ before the target onset. Trials that were wrongly responded to or with artifacts exceeding the amplitude of $\pm 75 \mu \mathrm{V}$ in any channel were rejected. After statistical evaluation, event-related potentials (ERPs) were, for presentation purposes only, low-pass filtered at $11 \mathrm{~Hz}$ (41 points, FIR).

Based on previous research on the N400 (see Koelsch, 2011 for a review), the time window from 300 to $500 \mathrm{msec}$ after the onset of target stimulus was selected for statistical analysis. Based on visual inspection and previous research on the N400 (Steinbeis \& Koelsch, 2008, 2011), electrodes were grouped into four separate regions of interest (ROIs): left anterior (AF3, F1, F3, F5, FC1, FC3, FC5), right anterior (AF4, F2, F4, F6, FC2, FC4, FC6), left posterior (PO3, P1, P3, P5, PO1, PO3, PO5), and right posterior (PO4, P2, P4, P6, PO2, PO4, PO6). To test for specific patterns of scalp distribution, we examined the effects of anteriority (anterior versus posterior ROIs) and hemisphere (left versus right ROIs) on ERP results. 
Repeated measures ANOVAs were conducted, with group (amusics, controls) as a between-subjects factor, and prime (happy, sad), target (happy, sad), anteriority (anterior, posterior), and hemisphere (left, right) as within-subjects factors. The mean of the respective electrodes in each region of interest was computed for analysis. To examine the effect of prime valence on target processing, the factors prime (happy, sad) and target (happy, sad) were entered into the analysis separately. A significant interaction between prime and target would indicate an affective priming effect, with congruous and incongruous targets showing different ERPs. As the main goal of this study was to examine the impact of amusia on the affective priming effect (i.e., the interaction between the prime and target), only the significant effects containing the main experimental variables (prime, target, and group) were reported (Goerlich et al., 2012; Steinbeis \& Koelsch, 2008, 2011). For illustration purposes, ERPs of congruous and incongruous trials were depicted without further differentiating the emotions of the prime/target.

\subsection{Results and discussion}

\subsubsection{Behavioral results}

The analysis of performance accuracy revealed an average score of $96.40 \%$ on the happiness/sadness judgment of the faces (amusics: $M=95.96 \%, S D=2.85$; controls: $M=$ $96.88 \%, S D=2.73$ ). A repeated measures ANOVA showed no significant main effects or interactions $(p s>.12)$. The major/minor triads had no priming effect on the accuracy of judgment of happiness/sadness of facial expressions, which may be attributed to the ceiling 
performance by both groups. Thus, accuracy scores did not seem to be sensitive to the prime-target relationship in the present experiment.

Figure 2 shows the mean reaction times ( \pm 1 SEM) for the judgment of happy versus sad facial expressions in controls and amusics, respectively. The factors prime and target were entered into a repeated measures ANOVA as within-subjects factors and group as a between-subjects factor. There was a main effect of group, $F_{(1,30)}=17.09, p<.001$, partial $\eta^{2}$ $=.36$, as amusics showed longer reaction times $(M=697.19, S D=83.48)$ than controls $(M=$ 577.29, $S D=80.61$ ) in judging happiness/sadness of the facial expressions. Moreover, the three-way interaction among prime, target, and group was significant, $F_{(1,30)}=6.34, p=.02$, partial $\eta^{2}=.17$. Further within-group analyses were conducted for amusics and controls. For controls, the interaction between prime and target was significant, $F_{(1,15)}=6.68, p=.02$, partial $\eta^{2}=.31$, suggesting that controls evaluated affectively congruous target images faster than affectively incongruous target images (see Figure 2). Results from the simple effects tests showed that this effect was present in controls only when the prime was happy $\left[F_{(1,15)}=\right.$ 9.05, $p=.009$, partial $\left.\eta^{2}=.38\right]$ but not when the prime was sad $\left[F_{(1,15)}=1.17, p=.30\right]$. For amusics, however, no significant main effect or interaction was observed ( $p \mathrm{~s}>.44)$, suggesting that happy/sad chords varying in major/minor mode did not prime the processing of happy/sad facial images in amusics.

Insert Figure 2, about here. 
Correlation analysis showed that neither accuracy nor reaction time was significantly correlated with the global MBEA score, pitch detection threshold, or pitch direction discrimination threshold for amusics or controls (all $p \mathrm{~s}>.09$ ).

\subsubsection{Electrophysiological results}

Analysis of the ERPs in the time window of $300-500 \mathrm{msec}$ revealed a significant main effect of target, as happy images elicited larger ERP amplitudes than sad images, $F_{(1,30)}=$ $4.88, p=.04$, partial $\eta^{2}=.14$. Moreover, there was a three-way interaction among prime, target, and group, $F_{(1,30)}=18.54, p<.001$, partial $\eta^{2}=.38$. Further within-group analyses were conducted for amusics and controls. For controls, the interaction between prime and target was significant, $F_{(1,15)}=15.97, p=.001$, partial $\eta^{2}=.52$, indicating a larger N400 for incongruous trials compared to congruous trials in controls (see Figure 3). Results from the simple effects tests showed that this N400 effect was present in controls only when the prime was happy $\left[F_{(1,15)}=25.68, p<.001\right.$, partial $\left.\eta^{2}=.63\right]$ but not when the prime was sad $\left[F_{(1,15)}\right.$ $=1.62, p=.22]$. For amusics, however, no significant main effect or interaction was observed ( $p$ s $>.23$ ), suggesting that happy/sad chords varying in major/minor mode did not prime the processing of happy/sad facial targets in amusics. No other significant main effects or interactions were observed ( $p \mathrm{~s}>.06)$.

Insert Figure 3, about here. 
The EEG results demonstrated that incongruent targets did not elicit a larger N400 compared to congruent targets in the judgment of emotional faces for amusics. These results were consistent with the lack of behavioral priming effect in terms of reaction time in amusics, indicating that emotional connotations of major/minor triads do not prime amusics' processing of emotional facial expressions. However, for controls, incongruent trials elicited a larger N400 than congruent trials over the majority of scalp regions. This electrophysiological effect was accompanied by the behavioral priming effect in terms of reaction time for controls. However, it is worth noting that only major chords, but not minor chords, primed the judgment of happy/sad faces for controls. This result might be due to the small number of trials included in the experiment ( 24 trials per condition). The findings also suggest that listeners may have higher emotional sensitivity to major than to minor chords. Future studies are needed to investigate these possibilities.

\section{Experiment 2: Automatic affective priming by consonant and}

\section{dissonant chords}

In Experiment 1, we used two consonant chords (major and minor triads) as primes. Detecting the difference between major and minor triads required subtle pitch interval analysis. Specifically, the intervals of a major or minor triad between the root and the fifth tones are always perfect fifth. The only difference between these two triads is the interval between the root and the third tones: One is major third while the other is minor third. From the perspective of music theory (Plomp \& Levelt, 1965), these consonant intervals consist of 
tones with simple frequency ratios, such as the perfect fifth (2:3) and the major third (4:5).

Unlike consonant intervals, dissonant intervals consist of tones with complex frequency ratios, such as the minor second (15:16), and the augmented fourth (i.e., the tritone, 32:45).

Therefore, the difference between consonant and dissonant chords may be perceived as more salient than that between two consonant chords.

Thus, the aim of Experiment 2 was to investigate whether isolated chords (consonant triads and dissonant non-triads) would prime affective representations of pleasant/unpleasant facial expressions in amusia. In order to create consonant versus dissonant chords, we manipulated the intervals between the root, the third, and the fifth tones of the chords. Following previous studies (e.g., Sollberge et al., 2003; Steinbeis \& Koelsch, 2011), the consonant stimuli in the present experiment contained major chords (e.g., C-E-G-C) in the root position and its inverted six-four chords, in which the intervals between the root and the third tones and those between the third and the fifth tones were always major and minor third. Dissonant stimuli involved two types of non-triads (e.g., C-F sharp-B-C and C-D flat-G flat-C), in which the intervals between the root and the third tones and those between the third and the fifth tones were not major or minor third but other intervals, such as minor second (15:16) and augmented fourth (32:45). Since the consonant stimuli had very simple frequency ratios and the dissonant stimuli had very complex frequency ratios, the chords in the present experiment were at the extreme ends of the consonance-dissonance distinction. We expected that the affective priming effect of consonant/dissonant chords on emotional 
recognition of facial expressions would be absent in amusics at both electrophysiological and behavioral levels as compared with controls.

\subsection{Method}

\subsubsection{Participants}

The participants were identical to those in Experiment 1.

\subsubsection{Stimuli}

Isolated chords were used as primes, and images of facial expressions were used as targets. The prime stimuli consisted of 48 chords with piano timbre, of which 24 were consonant (pleasant sounding) and 24 were dissonant (unpleasant sounding). Two types of chords were used for both consonant and dissonant stimuli, in which the root notes ranged from $\mathrm{G} 3$ to $\mathrm{F} \# 4$, resulting in 24 chords in each affective category. Chords were $800 \mathrm{msec}$ long, created using Cubase (Steinberg Media Technologies GmbH, Hamburg, Germany), exported with the piano timbre and modified with Audition 6.0 (sampling rate $=44.1 \mathrm{kHz} ; 16$-bit resolution).

The roughness and harmonicity (HNR) of the consonant and dissonant chords were calculated. The results showed that consonant chords had significantly higher HNRs than dissonant chords (consonant chords: $M=11.29, S D=8.76$; dissonant chords: $M=6.93, S D=$ 1.69; $z=-2.34, p=.018, r=-.34$ ), but the two did not differ in roughness (consonant chords: $M=45.13, S D=7.24$; dissonant chords: $M=45.71, S D=8.83 ; z=-0.03, p=.989, r<-.01)$. 
In order to assess the emotional connotations of these chords, eight non-musicians who did not participate in the subsequent ERP experiment were asked to rate each chord with regard to perceived pleasantness on a 7 -point scale $(7=$ very pleasant to $1=$ very unpleasant $)$. A paired samples $t$-test showed that the ratings of consonant chords $(M=4.77, S D=1.49)$ were significantly higher than dissonant chords $(M=3.88, S D=1.63)$ in terms of perceived pleasantness $\left[t_{(7)}=3.45, p=.01\right]$. This confirmed that the consonant and dissonant chords were different in pleasantness.

We chose 24 pleasant and 24 unpleasant images from the CFAPS (Gong et al., 2011) as the target stimuli, which were different from those used in Experiment 1. Similar to the happy images used in Experiment 1, the 24 pleasant images were represented by happy smiling faces in this experiment. Previous research has indicated that the affective priming effect can be modulated by stimuli with different arousal levels (Zhang et al., 2012). Given that happiness is characterized by high valence and high arousal, we chose 24 angry or scared faces (both with low valence and high arousal) as unpleasant images so as to control for the arousal level of pleasant (happy) and unpleasant (angry/scared) stimuli. Each chord was played twice, once followed by a congruous target image, and the second time by an incongruous target image. This resulted in four experimental conditions: congruent and incongruent conditions for pleasant chords as well as congruent and incongruent conditions for unpleasant chords (see Figure 4). There were 96 trials in total, with 24 pleasant congruent trials (pleasant chords and pleasant faces), 24 pleasant incongruent trials (pleasant chords and 
unpleasant faces), 24 unpleasant congruent trials (unpleasant chords and unpleasant faces), and 24 unpleasant incongruent trials (unpleasant chords and pleasant faces).

Insert Figure 4, about here.

\subsubsection{Procedure}

The 96 trials were presented pseudorandomly in two blocks of 48 trials. Chords were presented from two loudspeakers positioned to the left and right of the participant. Visual targets appeared $200 \mathrm{msec}$ following the onset of the chord on the screen. Participants were instructed to decide as quickly and accurately as possible whether the facial expression was pleasant or unpleasant by pressing one of the two response buttons. The side (left or right hand) of the response was balanced across participants. The first block began with a practice session of two trials to familiarize the participants with the procedure. ERP recording and data analysis were the same as in Experiment 1. The order of Experiments 1 and 2 was counterbalanced across participants.

\subsection{Results and discussion}

\subsubsection{Behavioral results}

The analysis of performance accuracy revealed an average score of $95.66 \%$ on the pleasantness judgment of the faces (amusics: $M=94.79 \%, S D=4.04$; controls: $M=96.60 \%$, 
$S D=2.06)$. A repeated measures ANOVA showed no significant main effects or interactions ( $p$ s $>.11$ ), which might be due to ceiling performance by both groups. Thus, given that the task was relatively easy, accuracy might not have been sensitive to the congruency of the prime-target pairs.

A repeated measures ANOVA was conducted on reaction times, with prime and target as within-subjects factors and group as a between-subjects factor. There was a main effect of group, $F_{(1,30)}=14.33, p=.001$, partial $\eta^{2}=.32$, with amusics $(M=711.11 \mathrm{msec}, S D=81.29)$ showing longer reaction times in judging the pleasantness of the faces than controls $(M=$ $599.50 \mathrm{msec}, S D=85.62$ ). Moreover, there was a three-way interaction among prime, target, and group, $F_{(1,30)}=9.21, p=.005$, partial $\eta^{2}=.24$. Further within-group analyses revealed a significant interaction between prime and target in controls, $F_{(1,15)}=15.97, p=.001$, partial $\eta^{2}=.52$, as controls judged the pleasantness of affectively congruous target images faster than affectively incongruous target images (see Figure 5). Results from the simple effects tests showed that this effect was present in controls only when the prime was pleasant $\left[F_{(1,15)}\right.$ $=20.45, p=.001$, partial $\left.\eta^{2}=.58\right]$ but not when the prime was unpleasant $\left[F_{(1,15)}=2.67, p\right.$ $=.123$, partial $\left.\eta^{2}=.15\right]$. For amusics, however, no significant main effect or interaction was observed $(p \mathrm{~s}>.11)$, which suggests that pleasant/unpleasant chords varying in consonance/dissonance did not prime amusics' processing of pleasant/unpleasant facial targets.

Insert Figure 5, about here. 
Correlation analysis showed that neither accuracy nor reaction time was significantly correlated with the global MBEA score, pitch detection threshold, or pitch direction discrimination threshold for amusics and controls (all $p \mathrm{~s}>.14$ ).

\subsubsection{Electrophysiological results}

Analysis of the ERPs in the time window of 300-500 msec revealed a significant three-way interaction between prime, target, and group, $F_{(1,30)}=18.54, p<.001$, partial $\eta^{2}$ $=.38$. For controls, the interaction between prime and target was significant, $F_{(1,15)}=15.97, p$ $=.001$, partial $\eta^{2}=.52$, indicating that controls exhibited a larger N400 for incongruous target images than for congruous target images (see Figure 6). Results from the simple effects tests showed that this $\mathrm{N} 400$ effect was present in controls both when the prime was pleasant $\left[F_{(1,15)}\right.$ $=12.25, p=.003$, partial $\left.\eta^{2}=.45\right]$ and when the prime was unpleasant $\left[F_{(1,15)}=5.26, p\right.$ $=.037$, partial $\left.\eta^{2}=.26\right]$. For amusics, however, no significant main effects or interactions were observed $(p s>.30)$, suggesting that pleasant/unpleasant chords varying in consonance/dissonance did not prime the processing of pleasant/unpleasant facial targets in amusics.

Insert Figure 6, about here.

There was a significant three-way interaction among anteriority, hemisphere, and group, $F_{(1,30)}=5.31, p=.03$, partial $\eta^{2}=.15$. For amusics, there was a main effect of anteriority, $F_{(1,}$ 
15) $=48.84, p<.001$, partial $\eta^{2}=.77$, indicating that the $\mathrm{N} 400$ amplitudes were larger in the posterior than the anterior region in amusics. For controls, there were main effects of anteriority, $F_{(1,15)}=48.84, p<.001$, partial $\eta^{2}=.77$, and hemisphere, $F_{(1,15)}=4.98, p=.04$, partial $\eta^{2}=.25$, as the $\mathrm{N} 400$ amplitudes of controls were larger in the posterior region and the left hemisphere, compared to the anterior region and the right hemisphere. Therefore, the observed three-way interaction might be due to a slight left weighting in controls relative to amusics. Other main effects or interactions were not significant $(p s>.06)$.

The results of Experiment 2 demonstrated that, for controls, incongruent trials elicited a larger N400 than congruent trials over the majority of scalp regions, suggesting that pleasant/unpleasant chords varying in consonance/dissonance primed the processing of pleasant/unpleasant facial targets in controls. However, unlike in Experiment 1 where controls only exhibited the N400 effect for positive emotions, the N400 effects in the present experiment were observed for both positive and negative emotions in controls. This might be because affective priming was more effective for high-arousal targets (happy, angry, and scared faces) than for low-arousal targets (sad faces) (Zhang et al., 2012). For amusics, incongruent trials did not elicit a larger N400 compared to congruent trials, suggesting a lack of priming effect of consonant/dissonant chords on the judgment of pleasant/unpleasant facial expressions in amusics. These EEG results were consistent with the behavioral priming effect, in which controls but not amusics showed faster reaction times to congruent versus incongruent trials. Overall, these findings suggest that emotional connotations of 
consonant/dissonant chords do not prime the processing of pleasant/unpleasant facial expressions for amusics.

\section{Experiment 3: Conscious emotional evaluation of isolated}

\section{chords}

Although Experiments 1 and 2 have shown that amusics are impaired in automatic processing of the emotional connotations of major/minor and consonant/dissonant chords, the results do not imply that they are also impaired in conscious evaluation of the emotions embedded in those chords. Therefore, the question remains as to whether conscious emotional processing of these chords is also impaired in amusia, given that automatic and conscious processing recruit distinct neural networks (e.g., Rossell et al., 2003; Gold et al., 2006). To answer this question, the present experiment was conducted to examine conscious emotional evaluation of major/minor chords in Task 1 and consonant/dissonant chords in Task 2. Given that previous studies have reported amusics' abnormal performance in judging musical emotions conveyed by major/minor mode (Gosselin et al., 2015) and consonant/dissonant chords (Cousineau et al., 2012), we expected that amusics in the present experiment would also exhibit impairments in conscious emotional evaluation of major/minor and consonant/dissonant chords as compared with controls. 


\subsection{Method}

\subsubsection{Participants}

Twelve amusics and 12 matched controls took part in this experiment. The recruitment and inclusion criteria of the participants were the same as in Experiments 1 and 2. Table 2 shows the demographic information, the mean scores of the MBEA, and the pitch discrimination thresholds of the participants. IQ was tested using the Combined Raven's Test (Wang et al., 2007), which included 72 items and was based on Raven's standard progressive matrix (Raven, 1960) and Raven's coloured progressive matrix (Raven, 1958). Working memory was tested using digit span tasks (forward and backward). As can be seen, the two groups differed significantly in the scores of the MBEA and in pitch thresholds, but were matched on age, years of education, IQ, and working memory. All participants were native speakers of Mandarin, right-handed, with normal hearing. None of them had received any extracurricular training in music. Ethical approval was obtained from Shanghai Normal University, and all participants signed a written consent form before the experiments were conducted.

Insert Table 2, about here. 


\subsubsection{Stimuli and procedure}

There were two tasks in the present experiment. Task 1 included the chords used in Experiment 1 (24 major and 24 minor triads), and Task 2 included those used in Experiment 2 (24 consonant and 24 dissonant chords). The two tasks were presented in separate blocks in a counterbalanced order. They were presented through two loudspeakers positioned to the left and right of the participant. For Task 1, participants were required to rate the happiness/sadness of each major/minor chord on a 7 -point scale $(7=$ very happy to $1=$ very sad) after the presentation of each chord. For Task 2, however, they were required to rate the pleasantness/unpleasantness of each consonant/dissonant chord using a 7-point scale $(7=$ very pleasant to $1=$ very unpleasant). For each task, there was a practice session of two trials to familiarize the participants with the procedure.

\subsection{Results and discussion}

Figure 7 shows the mean happiness/sadness rating ( \pm 1 SEM) of the major/minor chords in controls and amusics, respectively. The results showed a main effect of chord type, $F_{(1,22)}$ $=15.43, p=.001$, partial $\eta^{2}=.41$, as the rating of major triads was higher than that of minor triads. Moreover, there was a significant interaction between group and chord type, $F_{(1,22)}=$ $6.23, p=.02$, partial $\eta^{2}=.22$, owing to the fact that controls but not amusics differentiated the emotions conveyed by major and minor triads (Controls: $F_{(1,22)}=20.63, p<.001$, partial $\eta^{2}=.48$; Amusics: $\left.F_{(1,22)}=1.03, p=.32\right)$. The main effect of group was not significant, $F_{(1,22)}$ $=0.009, p=.93$. 
Correlation analysis showed that, for controls, the conscious evaluation of major/minor triads was significantly correlated with the global MBEA score $\left(r_{(11)}=.66, p=.02\right)$, but it was not correlated with pitch detection threshold, or pitch direction discrimination threshold ( $p \mathrm{~s}>.34)$. For amusics, however, the conscious evaluation of major/minor triads was not correlated with the global MBEA score, pitch detection threshold, or pitch direction discrimination threshold (all $p \mathrm{~s}>.09)$.

Insert Figure 7, about here.

Figure 8 shows the mean pleasantness/unpleasantness ratings ( \pm 1 SEM) of the consonant/dissonant chords in controls and amusics, respectively. The results showed a main effect of chord type, $F_{(1,22)}=37.45, p<.001$, partial $\eta^{2}=.63$, as the rating of consonant chords was higher than that of dissonant chords. Moreover, there was a significant interaction between group and chord type, $F_{(1,22)}=14.04, p=.001$, partial $\eta^{2}=.39$, indicating that controls but not amusics differentiated the emotions conveyed by consonant and dissonant chords (Controls: $F_{(1,22)}=48.67, p<.001$, partial $\eta^{2}=.69$; Amusics: $\left.F_{(1,22)}=2.82, p=.11\right)$. The main effect of group was not significant, $F_{(1,22)}=0.32, p=.58$.

Correlation analysis showed that, for controls, the conscious evaluation of consonant/dissonant chords was significantly correlated with the global MBEA score $\left(r_{(11)}\right.$ $=.73, p=.007)$, but it was not correlated with pitch detection threshold or pitch direction discrimination threshold $(p \mathrm{~s}>.08)$. For amusics, however, the conscious evaluation of 
consonant/dissonant chords was not correlated with the global MBEA score, pitch detection threshold, or pitch direction discrimination threshold (all $p \mathrm{~s}>.33$ ).

Insert Figure 8, about here.

These results are consistent with previous studies (Cousineau et al., 2012; Marin et al., 2015), suggesting that amusics cannot use the cues of harmonicity to evaluate musical emotions of major/minor and consonant/dissonant chords consciously. These findings also imply that amusics' deficits in conscious emotional processing of major/minor and consonant/dissonant chords is unrelated to their deficits in pitch discrimination.

\section{General discussion}

The present study investigated automatic and conscious emotional processing of isolated chords in individuals with and without congenital amusia. Using a cross-modal affective priming paradigm, Experiments 1 and 2 investigated whether the emotional connotations of isolated chords would prime the behavioral and neural processing of emotional faces in amusia. The results from both experiments indicated that, for controls, major/minor and consonant/dissonant chords primed the processing of happy/sad and pleasant/unpleasant faces, as reflected by longer reaction times and larger N400 amplitudes for the incongruent than congruent trials. However, amusics failed to demonstrate such a priming effect in both behavior and EEG. Furthermore, the results of Experiment 3 demonstrated that amusics could 
not consciously differentiate the emotions conveyed by major/minor or consonant/dissonant chords, as compared with controls. These findings reveal amusics' deficits in automatic and conscious processing of musical emotions conveyed by different pitch constructions in isolated chords.

The main finding of the present study is that amusics are impaired in automatic emotional processing of isolated chords. Unlike controls, amusics did not exhibit longer reaction times and larger N400 amplitudes for incongruent than congruent trials. Given that the N400 effect reflects the processing of affective meaning (Kutas \& Federmeier, 2011) through spreading activation (Collins \& Loftus, 1975; Shiffrin \& Schneider, 1977), the absence of the N400 effect in amusia indicates that single chords with emotional connotations do not automatically activate neural representations of emotional faces for amusics. Together with the response time data, the absence of electrophysiological effects in amusics indicated that emotional connotations of single chords could not automatically prime amusics' processing of emotional facial expressions.

The impairment in automatic emotional processing of chords in amusia may be due to amusics' cortical and subcortical anomalies in early auditory encoding. Indeed, previous neurophysiological studies have suggested that amusia is associated with impaired automatic processing of fine-grained sounds, with abnormal early cortical responses of N100m (Albouy et al., 2013; see also Jiang et al., 2012; Omigie et al., 2013) and subcortical anomalies in the auditory brainstem (Lehmann et al., 2015; but see Liu et al., 2015). These impairments may have a cascade effect on amusics' ability to encode rapid pitch information (Albouy et al., 
2016). Therefore, the failure to show the affective priming effect of musical chords on emotional faces in amusics may be attributed to their impaired encoding of pitch in the early processing stage. Given that the perception of musical emotions represents high-level processing of pitch information, the present findings imply that amusics' deficits may be relevant to automatic processing at both low and high levels of pitch processing, and there may be a neural network that is specific to pitch processing in music (Norman-Haignere et al., 2016; Zhou et al., 2017).

Several ERP studies have suggested that early cortical responses in amusia might be preserved under unconscious conditions (Zendel et al., 2015; see also Peretz et al., 2009). For example, Zendel et al. (2015) showed that amusics elicited a normal early right anterior negativity (ERAN) for out-of-tune and out-of-key notes in the unconscious but not in the conscious condition, although a recent study by Sun et al. (2018) showed that the ERAN was absent in the amusic group during the implicit (unconscious) task. It is worth noting that the elicitation of the ERAN relies on long-term learned knowledge of syntactic regularities in music (Koelsch, 2012; Trainor, 2012). Such knowledge is based on prior musical exposure and perceptual learning (Jiang et al., 2017; Wong et al., 2009). Unlike syntactic processing, however, automatic emotional processing may be equipped with a biological mechanism in humans (e.g., Bargh, 1989; Öhman, 1988; Öhman et al., 2000). Indeed, it has been shown that 2-month-old infants are capable of showing preference for consonant over dissonant musical intervals (Trainor et al., 2002). Therefore, the impaired automatic emotional processing of musical chords in amusia may suggest that basic auditory processes such as 
interval analysis are impaired in amusics. This also lends support to the hypothesis that congenital amusia is present from birth (Peretz et al., 2007).

Although Experiments 1 and 2 have shown that amusics are impaired in automatic emotional processing of chords, the results do not extend to conscious evaluation of musical emotions. Indeed, it has been suggested that early automatic and late conscious emotional processing may be dissociated (e.g., Castner et al., 2007; Kamio et al., 2006; Padovan et al., 2002; Suslow et al., 2003), owing to the fact that these two types of processing recruit distinct neural networks (e.g., Rossell et al., 2003; Gold et al., 2006). The results of Experiment 3 showed that, as compared with controls, amusics could not consciously perceive the difference in affect between major and minor and between consonant and dissonant chords. These findings are consistent with previous behavioral studies (Ayotte et al., 2002; Gosselin et al., 2015; Jiang et al., 2017), suggesting amusics' deficits in conscious emotional processing of pitch-related information. Such deficits may be due to the fact that amusics are unable to evaluate musical emotions based on the acoustic cues of harmonicity (Cousineau et al., 2012; Marin et al., 2015). Indeed, the major/minor and consonant/dissonant chords used in the present study differed in harmonicity but not in roughness. Taken together, our findings suggest that amusia may be associated with impairments in both automatic and conscious emotional processing of chords.

To conclude, our study provides the first electrophysiological evidence that amusics are impaired in automatic activation of emotional representations of single chords. Together with previous studies that show amusics' abnormal early neural responses to fine-grained pitch 
discrimination (Albouy et al., 2013; Jiang et al., 2012) and expectancy (Omigie et al., 2013), our findings imply that amusics' deficits may be related to automatic processing of musical pitch at both low and high levels of music processing. On the other hand, our findings also confirm amusics' deficits in conscious emotional evaluation of music, using the harmonicity cues in isolated chords. Taken together, the present study may provide insight into the neural origin of the anomaly in emotional processing in amusia. In particular, given that amusia is associated with subcortical anomalies in the auditory brainstem (Lehmann et al., 2015), and that the affective priming effect of chords is related to the activation of MTG and IFG (Steinbeis \& Koelsch, 2008), the absence of the affective priming effect in amusia may be due to abnormal cortical activities, subcortical anomalies in the auditory brainstem, or both. 


\section{Acknowledgements}

This work was supported by the National Natural Science Foundation of China (Grant No. 31470972 to C. J. and F. L., and Grant No. 31500876 to L. Z. and J. J.), and the European Research Council (ERC) Starting Grant to F. L. and C. J. (ERC-StG-2015, CAASD, 678733). We wish to thank the editor, Prof. Laurel Trainor, and two anonymous reviewers for their insightful comments and helpful suggestions on our earlier drafts. We also thank Can Zhou for her help with participant recruitment and data collection. 


\section{References}

Albouy, P., Cousineau, M., Caclin, A., Tillmann, B., \& Peretz, I. (2016). Impaired encoding of rapid pitch information underlies perception and memory deficits in congenital amusia. Scientific Reports, 6, 18861.

Albouy, P., Mattout, J., Bouet, R., Maby, E., Sanchez, G., Aguera, P.-E., . . Tillmann, B. (2013). Impaired pitch perception and memory in congenital amusia: The deficit starts in the auditory cortex. Brain, 136(5), 1639-1661.

Ayotte, J., Peretz, I., \& Hyde, K. (2002). Congenital amusia : A group study of adults afflicted with a music-specific disorder. Brain, 125(2), 238-251.

Bargh, J. A. (1989). Conditional automaticity: Varieties of automatic influence in social perception and cognition. In J. S. Uleman \& J. A. Bargh (Eds.), Unintended thought (pp. 3-51). New York, NY: Guilford Press.

Bargh, J.A., Chaiken, S., Govender, R., Pratto, F. (1992). The generality of the automatic attitude activation effect. Journal of Personality and Social Psychology, 62, 893-912.

Bargh, J.A., Chartrand, T.L. (1999). The unbearable automaticity of being. American Psychologist, 54, 462-479.

Brattico, E., Pallesen, K. J., Varyagina, O., Bailey, C., Anourova, I., Järvenpää, M., . . . Tervaniemi, M. (2009). Neural discrimination of nonprototypical chords in music experts and laymen: an MEG study. Journal of Cognitive Neuroscience, 21(11), 2230-2244. 
Castner, J. E., Chenery, H. J., Copland, D. A., Coyne, T. J., Sinclair, F., \& Silburn, P. A. (2007). Semantic and affective priming as a function of stimulation of the subthalamic nucleus in Parkinson's disease. Brain, 130(5), 1395-1407.

Collins, A. M., \& Loftus, E. F. (1975). A spreading-activation theory of semantic processing. Psychological Review, 82(6), 407-428.

Cousineau, M., McDermott, J. H., \& Peretz, I. (2012). The basis of musical consonance as revealed by congenital amusia. Proceedings of the National Academy of Sciences of the United States of America, 109(48), 19858-19863.

Fazio, R. H., Sanbonmatsu, D. M., Powell, M. C., \& Kardes, F. R. (1986). On the automatic activation of attitudes. Journal of Personality and Social Psychology, 50(2), 229-238.

Foxton, J. M., Dean, J. L., Gee, R., Peretz, I., \& Griffiths, T. D. (2004). Characterization of deficits in pitch perception underlying 'tone deafness'. Brain, 127(4), 801-810.

Gagnon, L., \& Peretz, I. (2003). Mode and tempo relative contributions to "happy-sad" judgements in equitone melodies. Cognition \& Emotion, 17(1), 25-40.

Goerlich, K. S., Witteman, J., Aleman, A., \& Martens, S. (2011). Hearing feelings: Affective categorization of music and speech in alexithymia, an ERP study. PLoS ONE, 6(5), e19501.

Goerlich, K. S., Witteman, J., Schiller, N. O., Van Heuven, V. J., Aleman, A., \& Martens, S. (2012). The nature of affective priming in music and speech. Journal of Cognitive Neuroscience, 24(8), 1725-1741. 
Gong, X., Huang, Y., Wang, Y., \& Luo, Y. (2011). Revision of the Chinese facial affective picture system. Chinese Mental Health Journal, 25(1), 40-46.

Gosselin, N., Paquette, S., \& Peretz, I. (2015). Sensitivity to musical emotions in congenital amusia. Cortex, 71, 171-182.

Henry, M. J., \& McAuley, J. D. (2013). Failure to apply signal detection theory to the Montreal Battery of Evaluation of Amusia may misdiagnose amusia. Music Perception, 30(5), $480-496$.

Hermans, D., De Houwer, J., \& Eelen, P. (2001). A time course analysis of the affective priming effect. Cognition \& Emotion, 15(2), 143-165.

Hermans, D., Houwer, J. D., \& Eelen, P. (1994). The affective priming effect: Automatic activation of evaluative information in memory. Cognition \& Emotion, 8(6), 515-533.

Herring, D. R., White, K. R., Jabeen, L. N., Hinojos, M., Terrazas, G., Reyes, S. M., . . Crites Jr, S. L. (2013). On the automatic activation of attitudes: A quarter century of evaluative priming research. Psychological Bulletin, 139(5), 1062.

Hevner, K. (1935). The affective character of the major and minor modes in music. The American Journal of Psychology, 47(1), 103-118.

Hyde, K. L., \& Peretz, I. (2004). Brains that are out of tune but in time. Psychological Science, 15(5), 356-360.

Jiang, C., Hamm, J. P., Lim, V. K., Kirk, I. J., Chen, X., \& Yang, Y. (2012). Amusia results in abnormal brain activity following inappropriate intonation during speech comprehension. PloS ONE, 7(7), e41411. 
Jiang, C., Hamm, J. P., Lim, V. K., Kirk, I. J., \& Yang, Y. (2010). Processing melodic contour and speech intonation in congenital amusics with Mandarin Chinese. Neuropsychologia, 48(9), 2630-2639.

Jiang, C., Hamm, J. P., Lim, V. K., Kirk, I. J., \& Yang, Y. (2011). Fine-grained pitch discrimination in congenital amusics with Mandarin Chinese. Music Perception, 28(5), $519-526$.

Jiang, C., Liu, F., \& Thompson, W. F. (2016). Impaired explicit processing of musical syntax and tonality in a group of Mandarin-speaking congenital amusics. Music Perception: An Interdisciplinary Journal, 33(4), 401-413.

Jiang, C., Liu, F., \& Wong, P. C. M. (2017). Sensitivity to musical emotion is influenced by tonal structure in congenital amusia. Scientific Reports. doi: $10.1038 / \mathrm{s} 41598-017-08005-\mathrm{X}$

Juslin, P. N., \& Laukka, P. (2004). Expression, perception, and induction of musical emotions: A review and a questionnaire study of everyday listening. Journal of New Music Research, 33(3), 217-238.

Kamio, Y., Wolf, J., Fein, D. J. J. o. A., \& Disorders, D. (2006). Automatic processing of emotional faces in high-functioning pervasive developmental disorders: An affective priming study. Journal of Autism and Developmental Disorders, 36(2), 155-167.

Kamiyama, K. S., Abla, D., Iwanaga, K., \& Okanoya, K. (2013). Interaction between musical emotion and facial expression as measured by event-related potentials. Neuropsychologia, 51(3), 500-505. 
Koelsch, S. (2012). Brain and Music. Wiley-Blackwell, Oxford, UK.

Koelsch, S. (2011). Towards a neural basis of processing musical semantics. Physics of Life Reviews, 8(2), 89-105.

Krumhansl, C. L. (2001). Cognitive foundations of musical pitch. New York: Oxford University Press.

Kutas, M., \& Federmeier, K. D. (2011). Thirty years and counting: Finding meaning in the N400 component of the event-related brain potential (ERP). Annual Review of Psychology, 62, 621-647.

Lehmann, A., Skoe, E., Moreau, P., Peretz, I., \& Kraus, N. (2015). Impairments in musical abilities reflected in the auditory brainstem: Evidence from congenital amusia. European Journal of Neuroscience, 42(1), 1644-1650.

Liu, F., Maggu, A. R., Lau, J. C., \& Wong, P. C. (2015). Brainstem encoding of speech and musical stimuli in congenital amusia: evidence from Cantonese speakers. Frontiers in Human Neuroscience, 8, 1029.

Liu, F., Patel, A. D., Fourcin, A., \& Stewart, L. (2010). Intonation processing in congenital amusia: Discrimination, identification and imitation. Brain, 133(6), 1682-1693.

Lolli, S., Lewenstein, A., Basurto, J., Winnik, S., \& Loui, P. (2015). Sound frequency affects speech emotion perception: Results from congenital amusia. Frontiers in Psychology, 6.

Loui, P., Guenther, F. H., Mathys, C., \& Schlaug, G. (2008). Action-perception mismatch in tone-deafness. Current Biology, 18(8), R331-R332. 
Marin, M. M., Thompson, W. F., Gingras, B., \& Stewart, L. (2015). Affective evaluation of simultaneous tone combinations in congenital amusia. Neuropsychologia, 78, 207-220.

McDermott, J. H., Lehr, A. J., \& Oxenham, A. J. (2010). Individual differences reveal the basis of consonance. Current Biology, 20(11), 1035-1041.

McDonald, C., \& Stewart, L. (2008). Uses and functions of music in congenital amusia. Music Perception, 25(4), 345-355.

Neely, J. H. (1977). Semantic priming and retrieval from lexical memory: Roles of inhibitionless spreading activation and limited-capacity attention. Journal of Experimental Psychology: General, 106, 226-254.

Norman-Haignere, S. V., Albouy, P., Caclin, A., McDermott, J. H., Kanwisher, N. G., \& Tillmann, B. (2016). Pitch-responsive cortical regions in congenital amusia. The Journal of Neuroscience, 36(10), 2986-2994.

Öhman, A. (1988). Preattentive processes in the generation of emotions. In V. Hamilton, G. H. Bower \& N. Frijda (Eds.), Cognitive perspectives on emotion and motivation (pp. 127-143). Dordrecht: Kluwer Academic.

Öhman, A., Flykt, A., \& Lundqvist, D. (2000). Unconscious emotion: Evolutionary perspectives, psychophysiological data and neuropsychological mechanisms. In R. D. Lane \& L. Nadel (Eds.), Cognitive neuroscience of emotion (pp. 296-327). New York: Oxford University Press.

Oldfield, R. C. (1971). The assessment and analysis of handedness: The Edinburgh inventory. Neuropsychologia, 9(1), 97-113. 
Omigie, D., Müllensiefen, D., Stewart, L. (2012). The experience of music in congenital amusia. Music Perception, 30, 1-18.

Omigie, D., Pearce, M. T., \& Stewart, L. (2012). Tracking of pitch probabilities in congenital amusia. Neuropsychologia, 50(7), 1483-1493.

Omigie, D., Pearce, M. T., Williamson, V. J., \& Stewart, L. (2013). Electrophysiological correlates of melodic processing in congenital amusia. Neuropsychologia, 51(9), $1749-1762$.

Padovan, C., Versace, R., Thomas-Antérion, C., \& Laurent, B. (2002). Evidence for a selective deficit in automatic activation of positive information in patients with Alzheimer's disease in an affective priming paradigm. Neuropsychologia, 40(3), 335-339.

Patel, A. D., Wong, M., Foxton, J., Lochy, A., \& Peretz, I. (2008). Speech intonation perception deficits in musical tone deafness (congenital amusia). Music Perception, $357-368$.

Peretz, I., Ayotte, J., Zatorre, R. J., Mehler, J., Ahad, P., Penhune, V. B., \& Jutras, B. (2002). Congenital amusia: A disorder of fine-grained pitch discrimination. Neuron, 33(2), $185-191$.

Peretz, I., Brattico, E., Järvenpää, M., \& Tervaniemi, M. (2009). The amusic brain: In tune, out of key, and unaware. Brain, 132(5), 1277-1286.

Peretz, I., Champod, A. S., \& Hyde, K. L. (2003). Varieties of musical disorders: The Montreal Battery of Evaluation of Amusia. Annals of the New York Academy of sciences, 999, $58-75$. 
Peretz, I., Cummings, S., \& Dubé, M.-P. (2007). The genetics of congenital amusia (tone deafness): A family-aggregation study. The American Journal of Human Genetics, 81(3), $582-588$

Pfeifer, J., \& Hamann, S. (2015). Revising the diagnosis of congenital amusia with the Montreal Battery of Evaluation of Amusia. Frontiers in Human Neuroscience, 9, 161.

Plomp, R., \& Levelt, W. J. M. (1965). Tonal consonance and critical bandwidth. Journal of the Acoustical Society of America, 38, 548-560.

Posner, M. I., \& Snyder, C. R. R. (1975). Attention and cognitive control. In R. L. Solso (Ed.), Information processing and cognition: The Loyola symposium (pp. 55-85). Hillsdale, NJ: Erlbaum.

Raven, J. C. (1958). Guide to using the soloured progressive matrices. London: HK Lewis.

Raven, J. C. (1960). Guide to the standard progressive matrices: Sets A, B, C, D and E. London: HK Lewis.

Scherer, K.R. (2001). Appraisal considered as a process of multilevel sequential checking. In: Scherer, K.R., Schorr, A., Johnstone, T. (Eds.), Appraisal processes in emotion: Theory, methods, research (pp. 92-120). Oxford University Press, Oxford.

Shiffrin, R. M., \& Schneider, W. (1977). Controlled and automatic human information processing: II. Perceptual learning, automatic attending and a general theory. Psychological Review, 84(2), 127-190.

Sollberge, B., Rebe, R., \& Eckstein, D. (2003). Musical chords as affective priming context in a word-evaluation task. Music Perception: An Interdisciplinary Journal, 20(3), 263-282. 
Steinbeis, N., \& Koelsch, S. (2008). Comparing the processing of music and language meaning using EEG and fMRI provides evidence for similar and distinct neural representations. PLoS ONE, 3(5), e2226.

Steinbeis, N., \& Koelsch, S. (2011). Affective priming effects of musical sounds on the processing of word meaning. Journal of Cognitive Neuroscience, 23(3), 604-621.

Suslow, T., Roestel, C., Droste, T., \& Arolt, V. (2003). Automatic processing of verbal emotion stimuli in schizophrenia. Psychiatry Research, 120(2), 131-144.

Sun, Y., Lu, X., Ho, H. T., Johnson, B. W., Sammler, D., \& Thompson, W. F. (2018). Syntactic processing in music and language: Parallel abnormalities observed in congenital amusia. NeuroImage: Clinical, 19, 640-651.

Thompson, W. F., Marin, M. M., \& Stewart, L. (2012). Reduced sensitivity to emotional prosody in congenital amusia rekindles the musical protolanguage hypothesis. Proceedings of the National Academy of Sciences of the United States of America, 109(46), 19027-19032.

Tillmann, B., Gosselin, N., Bigand, E., \& Peretz, I. (2012). Priming paradigm reveals harmonic structure processing in congenital amusia. Cortex, 48(8), 1073-1078.

Tillmann, B., Lalitte, P., Albouy, P., Caclin, A., \& Bigand, E. (2016). Discrimination of tonal and atonal music in congenital amusia: The advantage of implicit tasks. Neuropsychologia, 85, 10-18. 
Trainor, L. J. (2012). Predictive information processing is a fundamental learning mechanism present in early development: Evidence from infants. International Journal of Psychophysiology, 83(2), 256-258.

Trainor, L. J., Tsang, C. D., \& Cheung, V. H. W. (2002). Preference for sensory consonance in 2- and 4-month-old infants. Music Perception, 20(2), 187-194.

Vassilakis, P. (2001). Auditory roughness estimation of complex spectra—Roughness degrees and dissonance ratings of harmonic intervals revisited. The Journal of the Acoustical Society of America, 110(5), 2755-2755.

Vassilakis, P. N. (2005). Auditory roughness as means of musical expression. Selected Reports in Ethnomusicology, 12, 119-144.

Vassilakis, P. N., \& Fitz, K. (2007). SRA: A web-based research tool for spectral and roughness analysis of sound signals. Paper presented at the Proceedings of the 4th Sound and Music Computing (SMC) Conference.

Wang, D., Di, M., Qian, M. (2007). A report on the third revision of combined Raven's Test (CRT-C3) for children in China. Chinese Journal of Clinical Psychology,15, 559-568.

Wong, P. C., Roy, A. K., \& Margulis, E. H. (2009). Bimusicalism: The implicit dual enculturation of cognitive and affective systems. Music Perception, 27(2), 81-88.

Zendel, B. R., Lagrois, M.-É., Robitaille, N., \& Peretz, I. (2015). Attending to pitch information inhibits processing of pitch information: The curious case of amusia. The Journal of Neuroscience, 35(9), 3815-3824. 
Zhang, Q., Kong, L., \& Jiang, Y. (2012). The interaction of arousal and valence in affective priming: Behavioral and electrophysiological evidence. Brain Research, 1474, 60-72.

Zhou, L., Liu, F., Jing, X., \& Jiang, C. (2017). Neural differences between the processing of musical meaning conveyed by direction of pitch change and natural music in congenital amusia. Neuropsychologia, 96, 29-38. 


\section{Table}

Table 1. Participants' characteristics and mean scores of the MBEA for each group in

Experiments 1-2. Standard deviation values are shown in parentheses.

\begin{tabular}{llll}
\hline & $\begin{array}{l}\text { Amusic } \\
(\mathrm{n}=16)\end{array}$ & $\begin{array}{l}\text { Control } \\
(\mathrm{n}=16)\end{array}$ & t-Test \\
\hline Mean age & $24.25(2.21)$ & $23.38(2.19)$ & n.s. \\
Sex & $10 \mathrm{~F}, 6 \mathrm{M}$ & $12 \mathrm{~F}, 4 \mathrm{M}$ & \\
Years education & $17.06(2.02)$ & $16.94(2.21)$ & n.s. \\
Melodic score of MBEA & $18.52(2.05)$ & $27.46(1.05)$ & $p<.001$ \\
Global score of MBEA & $18.78(1.37)$ & $27.26(0.85)$ & $p<.001$ \\
d' of MBEA & $0.84(0.31)$ & $2.75(0.27)$ & $p<.001$ \\
Pitch detection threshold & $1.59(1.63)$ & $0.44(0.30)$ & $p=.0096$ \\
$\begin{array}{l}\text { Pitch direction discrimination } \\
\text { threshold }\end{array}$ & $4.45(1.70)$ & $2.32(2.32)$ & $p=.006$ \\
\hline
\end{tabular}

Note: $\mathrm{F}=$ female; $\mathrm{M}=$ male. The "melodic score of MBEA" refers to the mean score of the three pitch-based subtests. n.s., non-significant. 
Table 2. Participants' characteristics and mean scores of the MBEA and pitch thresholds for each group in Experiment 3. Standard deviation values are shown in parentheses.

\begin{tabular}{|c|c|c|c|}
\hline & $\begin{array}{l}\text { Amusic } \\
(n=12)\end{array}$ & $\begin{array}{l}\text { Control } \\
(n=12)\end{array}$ & t-Test \\
\hline Mean age & $22.58(0.67)$ & $22.91(0.79)$ & n.s. \\
\hline Sex & 9F, 3M & 9F, 3M & \\
\hline Years education & $15.92(0.67)$ & $16.08(0.51)$ & n.s. \\
\hline IQ & $67.67(1.78)$ & $66.83(4.34)$ & n.s. \\
\hline Forward digit span & $8.33(0.78)$ & $8.75(0.45)$ & n.s. \\
\hline Backward digit span & $5.92(1.98)$ & $7.08(1.56)$ & n.s. \\
\hline Melodic score of MBEA & $19.92(1.06)$ & $27.36(1.20)$ & $p<.001$ \\
\hline Global score of MBEA & $20.14(1.49)$ & $27.39(1.43)$ & $p<.001$ \\
\hline d' $^{\prime}$ of MBEA & $1.12(0.05)$ & $7.80(0.05)$ & $p<.001$ \\
\hline Pitch detection threshold & $1.23(0.69)$ & $0.45(0.46)$ & $p=.004$ \\
\hline Pitch direction discrimination threshold & $4.31(1.94)$ & $1.44(1.45)$ & $p<.001$ \\
\hline
\end{tabular}

Note: $\mathrm{F}=$ female; $\mathrm{M}=$ male. The "Melodic score of MBEA" refers to the mean score of the three pitch-based subtests. n.s., non-significant. 


\section{Figure captions}

Figure 1. Design of the cross-modal affective priming paradigm in Experiment 1. Happy/sad chords characterized by major/minor quality were used as primes, and happy/sad facial expression images were used as targets. Target images were affectively congruent or incongruent to the prime music. SOA: stimulus onset asynchrony.

Figure 2. Experiment 1: Mean reaction times $( \pm 1 \mathrm{SEM})$ in $(\mathrm{A})$ amusics and $(\mathrm{B})$ controls for judgement of happy/sad facial expressions.

Figure 3. Experiment 1: Grand mean waveforms elicited by congruent and incongruent target images at four electrode sites for amusics and controls, respectively. Bottom: Scalp distribution of the incongruent-minus-congruent difference waves in the 300-500 ms latency range for amusics and controls, respectively.

Figure 4. Design of the cross-modal affective priming paradigm in Experiment 2.

Pleasant/unpleasant chords characterized by consonant/dissonant quality were used as primes, and pleasant/unpleasant facial expression images were used as targets. Target images were affectively congruent or incongruent to the prime music.

Figure 5. Experiment 2: Mean reaction times ( \pm 1 SEM) in (A) amusics and (B) controls for judgment of pleasant/unpleasant facial expressions.

Figure 6. Experiment 2: Grand mean waveforms elicited by congruent and incongruent target images at four electrode sites for amusics and controls, respectively. Bottom: Scalp 
distribution of the incongruent-minus-congruent difference waves in the 300-500 ms latency range for amusics and controls, respectively.

Figure 7. Mean happiness/sadness ratings ( $\pm 1 \mathrm{SEM})$ of the major/minor chords in controls and amusics in Experiment 3, respectively. n.s., non-significant; *** $p<.001$.

Figure 8. Mean pleasantness/unpleasantness ratings ( $\pm 1 \mathrm{SEM})$ of the consonant/dissonant chords in controls and amusics in Experiment 3, respectively. n.s., non-significant; $* * * p$ $<.001$. 
Figure 1

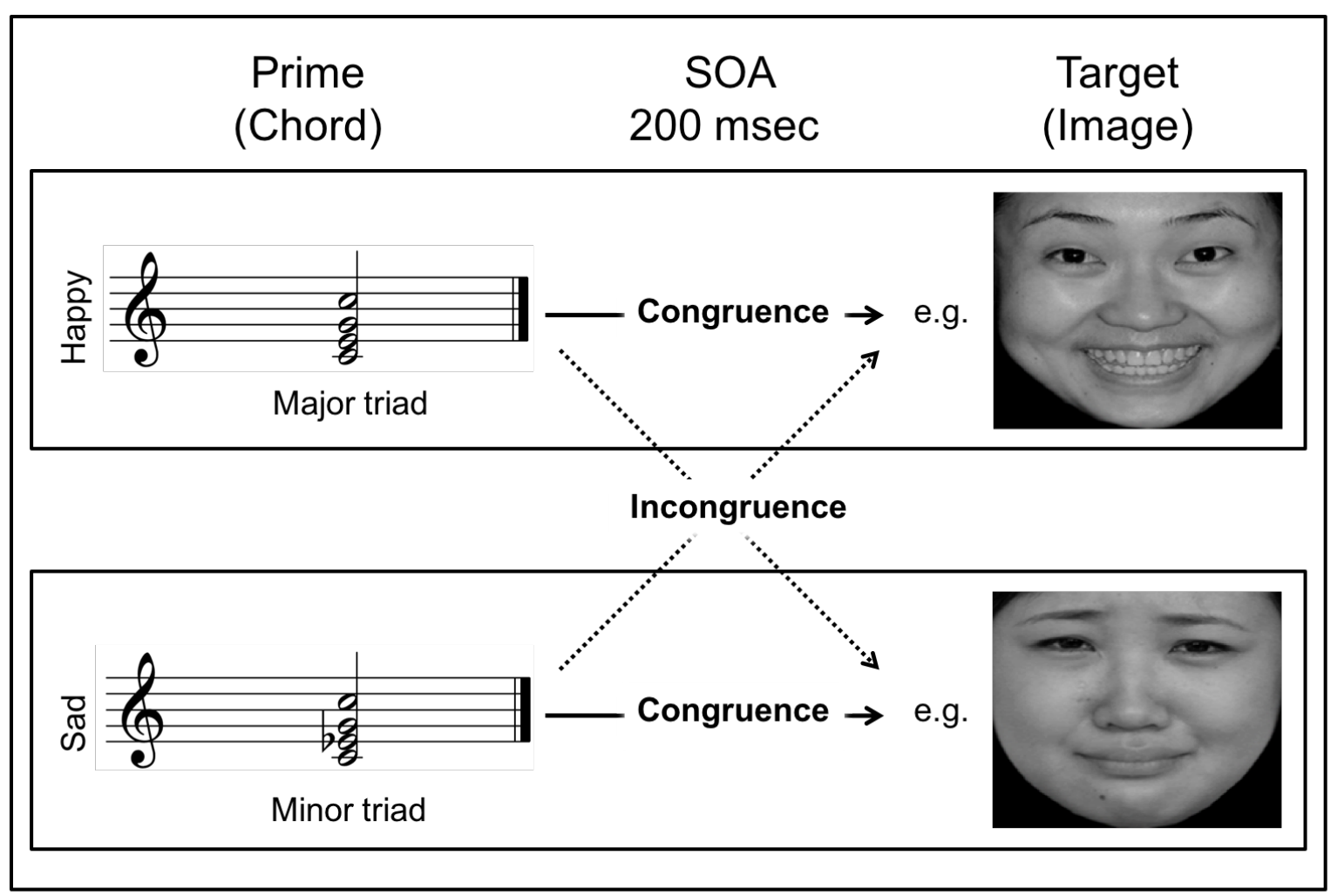


Figure 2
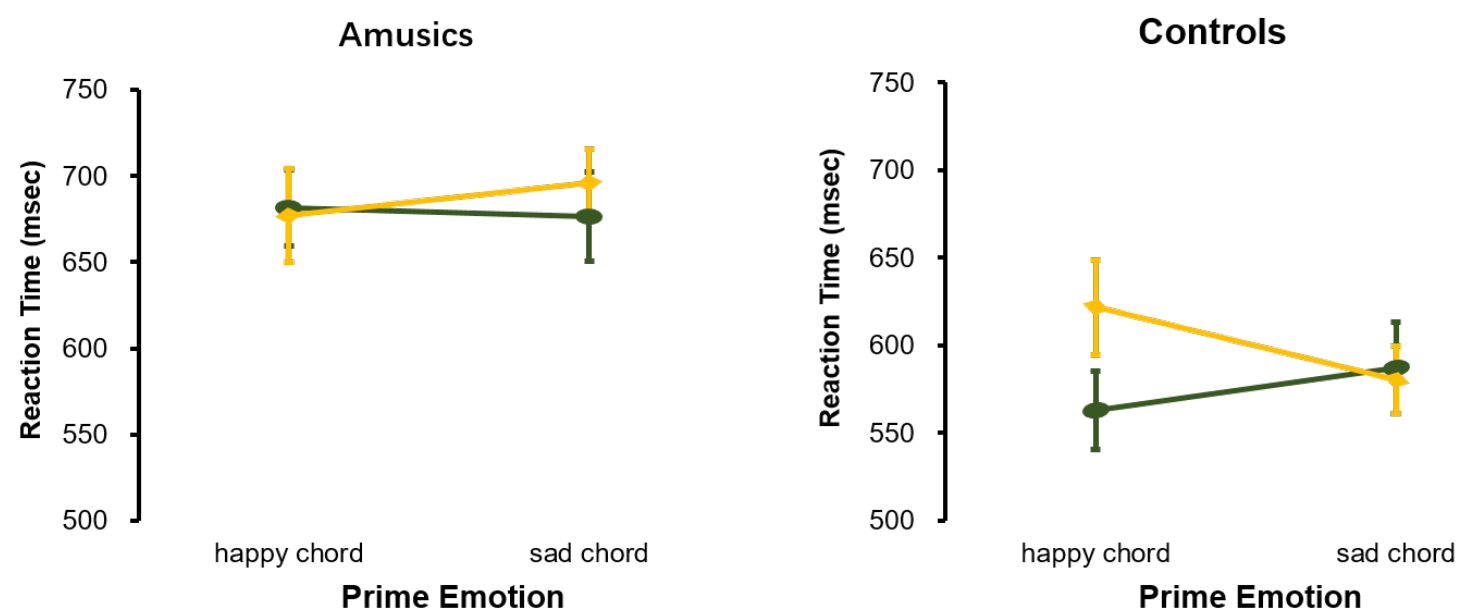

Prime Emotion

Prime Emotion

$\longrightarrow$ happy target image

sad target image 
Figure 3

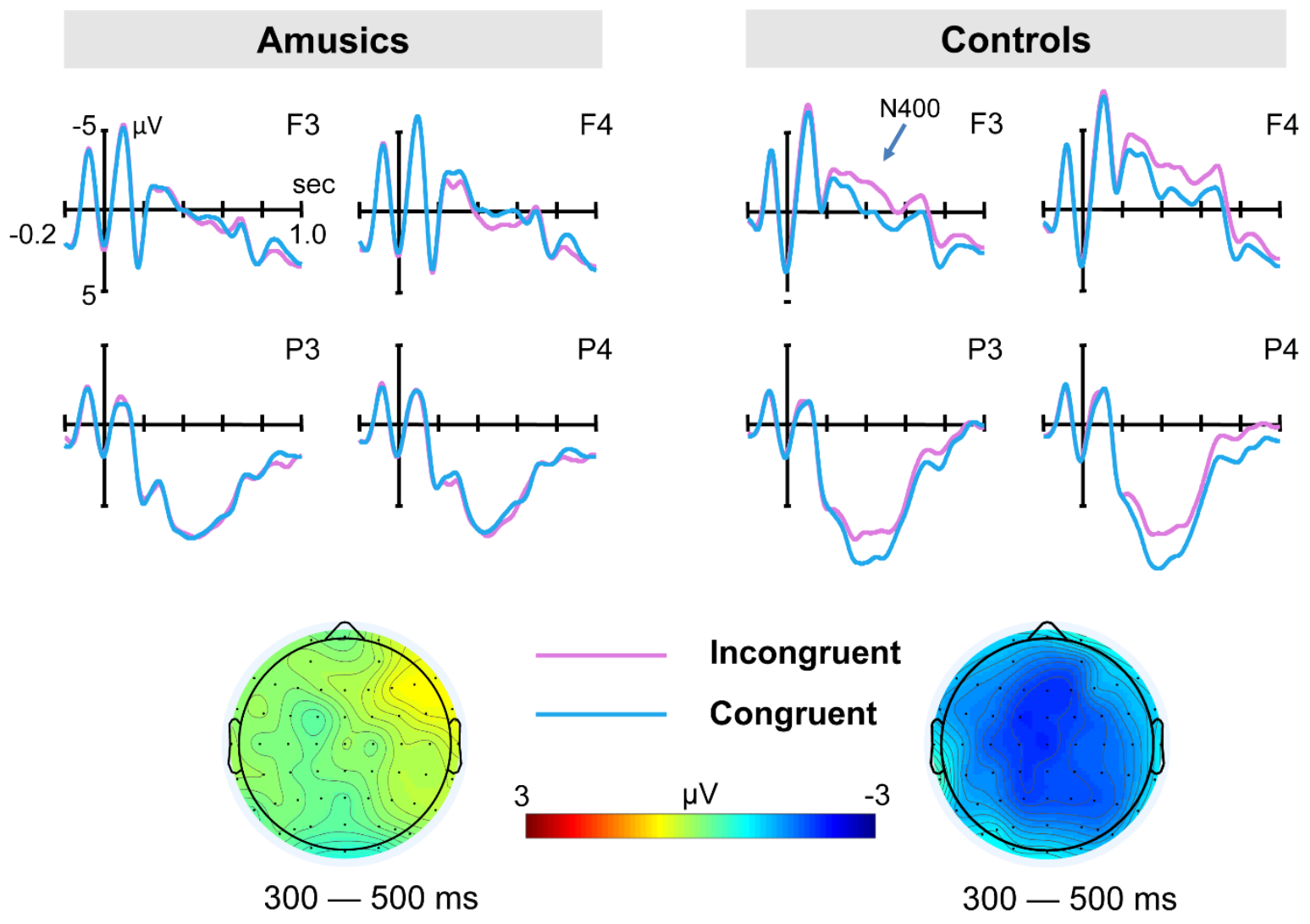


Figure 4

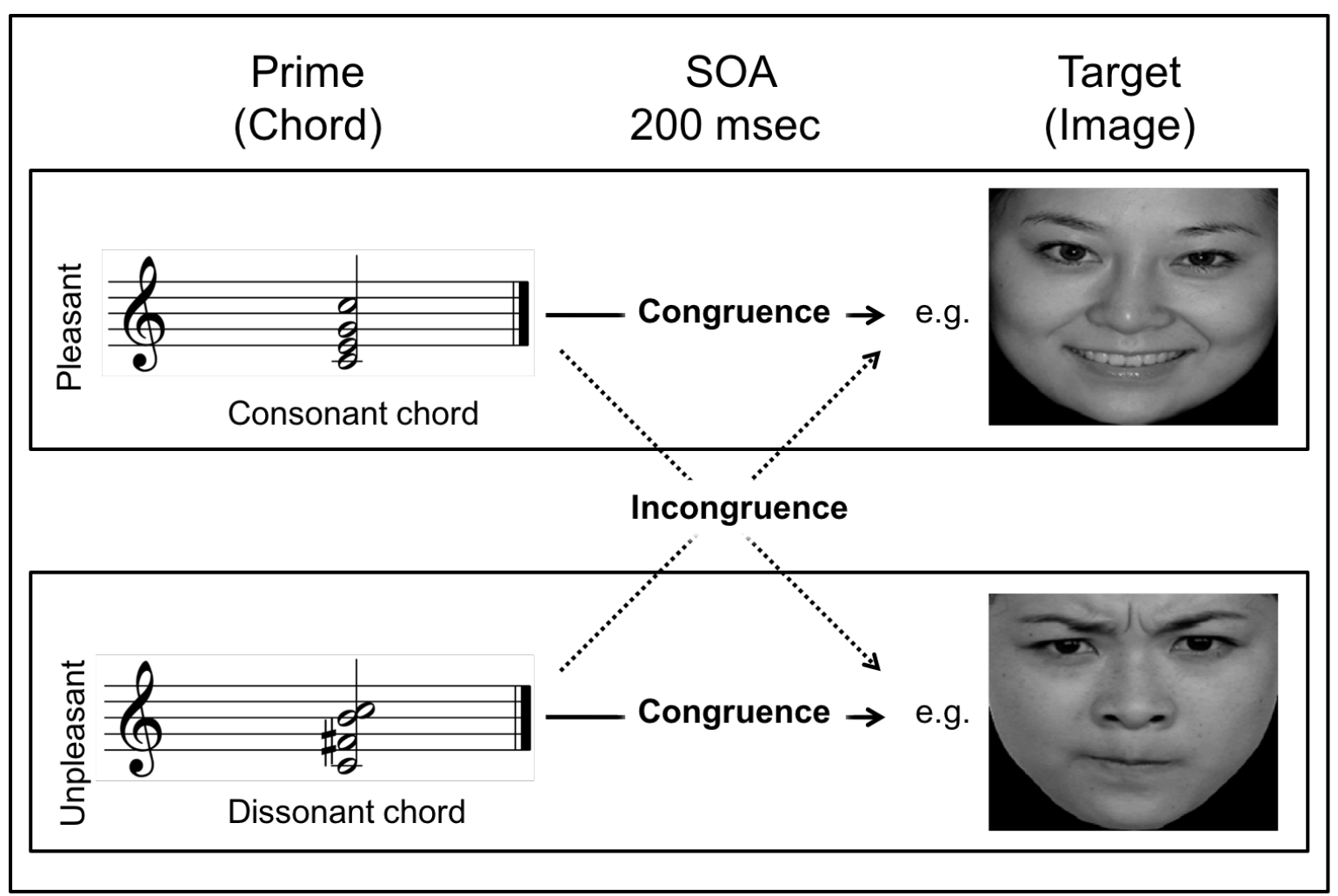


Figure 5

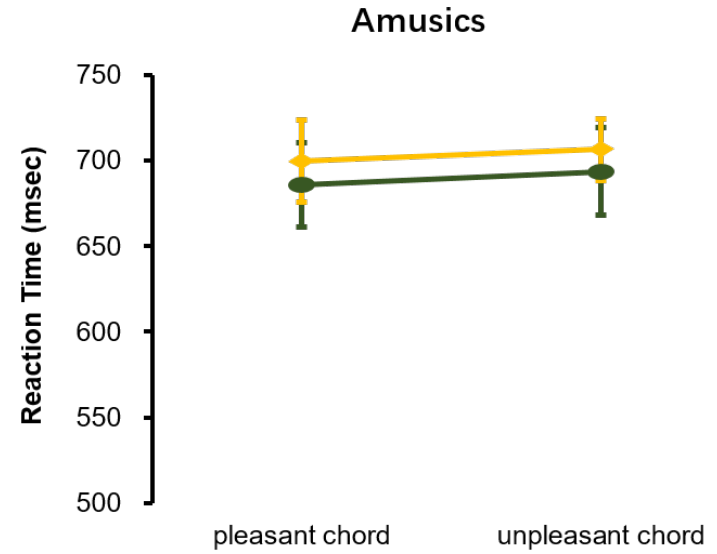

Prime Emotion
Controls

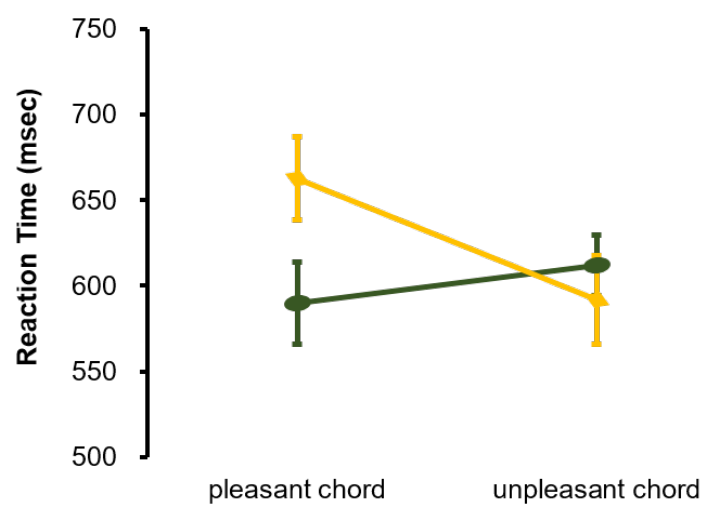

Prime Emotion

$\longrightarrow$ pleasant target image

unpleasant target image 
Figure 6

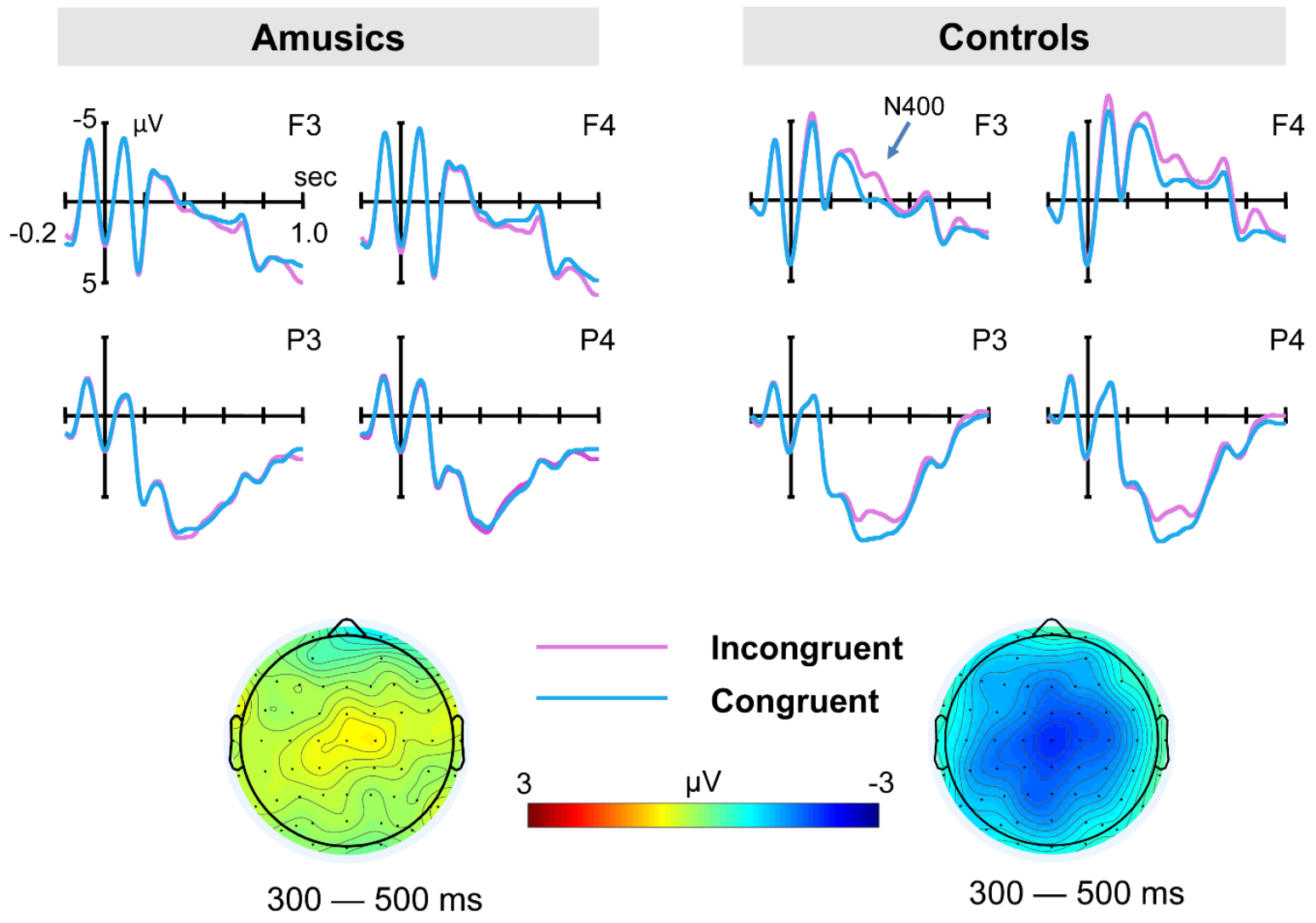


Figure 7

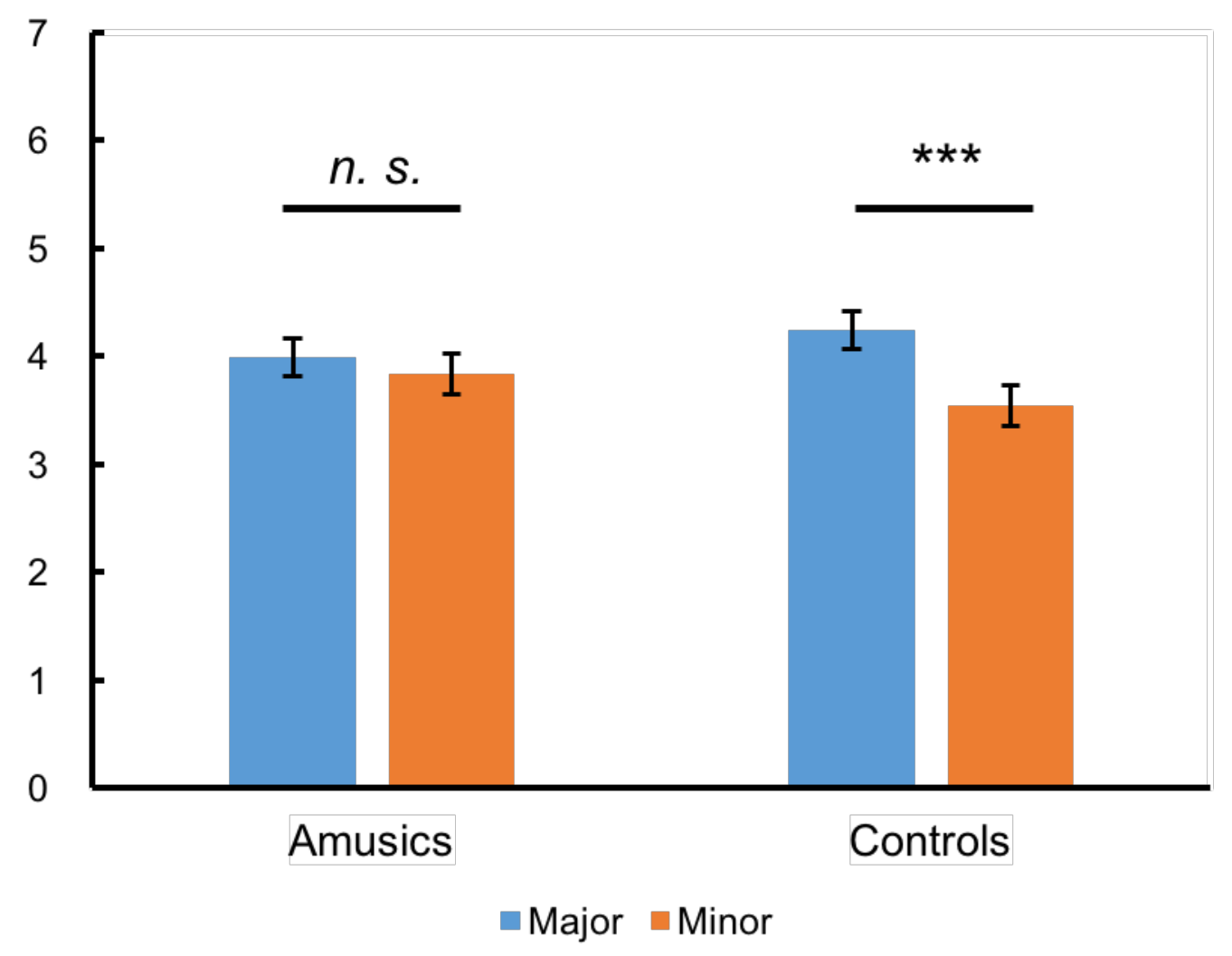


Figure 8

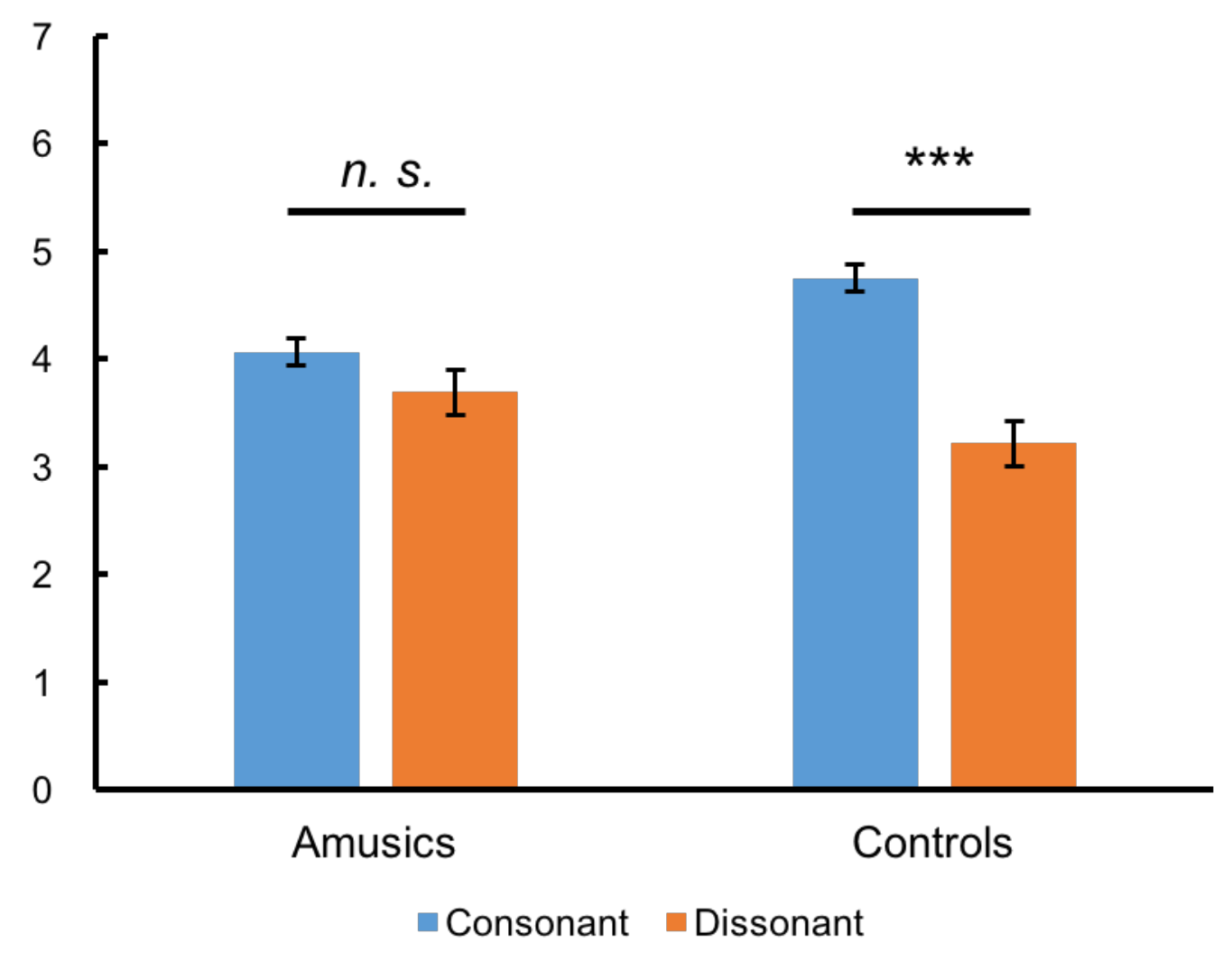

